International Journal of Wireless \& Mobile Networks (IJWMN) Vol. 5, No. 1, February 2013

\title{
Peak to Average Power Ratio Reduction of OFDM SIGNAL BY COMBINING CLIPPING WITH WALSH HADAMARD TRANSFORM
}

\author{
Navneet Kaur and Lavish Kansal \\ Department of Electronics and Communication Engineering \\ Lovely Professional University, Phagwara \\ Punjab, India \\ nv_neet@yahoo.com \\ Department of Electronics and Communication Engineering \\ Lovely Professional University, Phagwara \\ Punjab, India \\ lavish.15911@lpu.co.in
}

\begin{abstract}
Wireless communications have been developed widely and rapidly in the modern world especially during the last decade. Orthogonal Frequency Division Multiplexing (OFDM) has grown to a popular communication technique for high speed communication. Besides of the advantages, one of main disadvantage of OFDM is high peak to average power ratio (PAPR). In this paper, a PAPR reduction method is proposed that is based on combining clipping with Walsh Hadamard Transform (WHT).WHT is a precoding technique which is having less complexity compared to the other existing power reduction techniques and also it can reduce PAPR considerably and results in no distortion. The performance of the proposed scheme is examined through computer simulations and it is found that power reductions are obtained.
\end{abstract}

\section{KEYWORDS}

OFDM, PAPR, WHT, MC, DMT, FFT, PSK, QAM SLM, PTS, CCDF.

\section{INTRODUCTION}

Recent advances in wireless communication systems have increased the throughput over wireless channels. The reliability of wireless communication has also been increased. But still the bandwidth and spectral availability demands are endless. The need to achieve reliable wireless systems with high spectral efficiency, low complexity and good error performance results in continued research in this field. To provide such a high spectral efficiency, an efficient modulation scheme is to be employed [1,2]. A promising modulation technique that is increasingly being considered is Orthogonal Frequency Division Multiplexing (OFDM) due to its advantages in dealing with the multipath propagation problem, high data rate and bandwidth efficiency [3].Being an important member of the multicarrier modulation (MC) techniques, OFDM is also called Discrete Multitone Modulation (DMT). It is based upon the principle of frequency division multiplexing (FDM) where each frequency channel is modulated with simpler modulation scheme. It splits a high rate data stream into a number of lower rate streams that are transmitted simultaneously over a number of orthogonal subcarriers [4]. Orthogonality is achieved by ensuring that the carriers are placed exactly at the nulls in the modulation spectra of each other. The increase of symbol duration for the lower rate parallel subcarriers reduces the relative amount of dispersion in time caused by multipath delay spread.

DOI : 10.5121/ijwmn.2013.5103 
International Journal of Wireless \& Mobile Networks (IJWMN) Vol. 5, No. 1, February 2013

However, OFDM also has its shortcoming. The major drawback of OFDM signal is its large peak-to-average power ratio (PAPR), which causes poor power efficiency or serious performance degradation to transmit power amplifier. To reduce the PAPR, many techniques have been proposed. Such as clipping, partial transmit sequence (PTS) [5], selected mapping (SLM) [6], interleaving, nonlinear companding transforms, hadamard transforms [7] and other techniques etc. [8,9]. These schemes can mainly be categorized into signal scrambling techniques, such as PTS, and signal distortion techniques such as clipping. Among those PAPR reduction methods, the simplest scheme is to use the clipping process.

The organization of this paper is as follow. Section 2 presents OFDM signal model and formulates the problem of PAPR. Section 3, Walsh Hadamard Transform precoding is discussed. Existing clipping technique is described in section 4.Proposed scheme based on combined WHT transform and clipping technique is explained in section 5. Section 6 presents simulation results. Conclusions are drawn in section 7.

\section{OFDM SIGNAL MODEL AND PAPR PROBLEM}

\subsection{OFDM System}

The simulation model OFDM system is presented in figure 1. This model consists of a transmitter, a channel and a receiver.

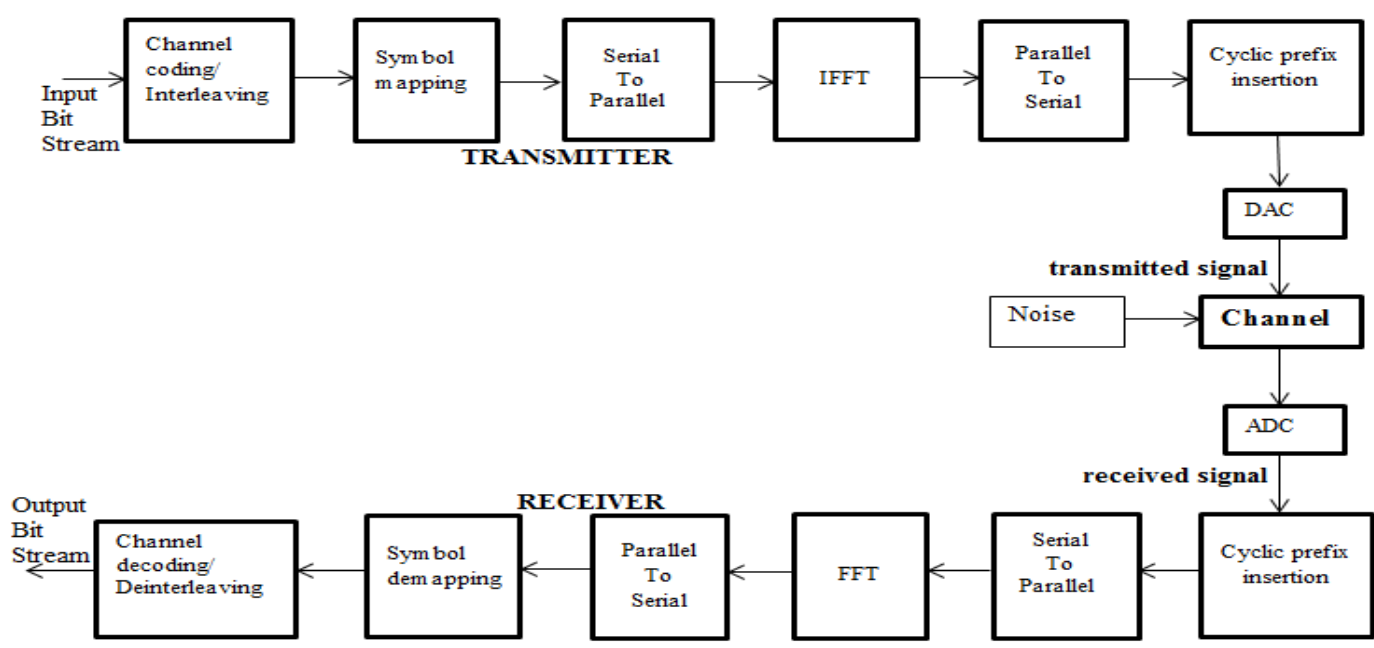

Figure 1. Block Diagram of OFDM system

In an OFDM scheme, a large number of orthogonal, overlapping, narrow band sub-carriers are transmitted in parallel each sub-carrier is then modulated with a conventional modulation scheme such as Quadrature Amplitude Modulation (QAM) or Phase-Shift Keying (PSK) at a low symbol rate than that required for the whole data stream, but still maintaining total data rates similar to conventional single-carrier modulation schemes in the same bandwidth. A highrate data stream is split into a number of lower rate streams to be transmitted simultaneously over a number of sub-carriers. Since the symbol duration increases for lower rate parallel subcarriers, the amount of dispersion in time caused due to multipath delay is reduced. These carriers divide the available transmission bandwidth. The separation of the sub-carriers is such that there is a very compact spectral utilization and each being modulated at a low bit rate. In a conventional frequency division multiplex the carriers are individually filtered to ensure there is no spectral overlap. 
International Journal of Wireless \& Mobile Networks (IJWMN) Vol. 5, No. 1, February 2013

\subsection{PAPR in OFDM System}

OFDM signal is expressed as a sum of independent subcarriers in the time and frequency directions and multiplied by the data symbols. An OFDM signal in baseband is defined as:

$$
s(t)=\sum_{n=0}^{N-1}\left(b_{n} e^{j 2 \pi f_{n} t} w(t)\right) ; 0 \leq t \leq T
$$

where, $b_{n}$ denotes the complex symbol modulating the $n$-th carrier, $w(t)$ is the time window function defined in the interval $[0, \mathrm{~T}], N$ is the number of subcarriers, and $T$ is the duration of an OFDM symbol. Subcarriers are spaced $\Delta f=1 / T$ apart. Each subcarrier is located at:

$$
f=\frac{n}{T} ; 0<n<N-1
$$

In order to maintain the orthogonality between the OFDM symbols, the symbol duration and sub channel space must meet the condition $T \Delta f=1$. Presence of large number of independently modulated subcarriers in an OFDM system the peak value of the system can be very high as compared to the average of the whole system. This ratio of the peak to average power value is termed as Peak-to-Average Power Ratio. Coherent addition of $N$ signals of same phase produces a peak which is $N$ times the average signal. High PAPR increases the complexity in the analog to digital and digital to analog converter and reduces the efficiency of RF amplifiers. In OFDM, a block of $N$ symbols $\left\{b_{n}, n=0,1, \ldots, N-1\right\}$ is formed with each symbol modulating one of a set of subcarriers; $\{f, n=0,1, \ldots, N-1\}^{n}$. The resulting signal is given as:

$$
s(t)=\frac{1}{\sqrt{N}} \sum_{n=0}^{N-1} b_{n} e^{j 2 \pi \Delta f t} ; 0 \leq t \leq N T
$$

The PAPR of the transmitted signal is defined as:

$$
P A P R=\frac{\max |s(t)|^{2}}{\frac{1}{N T} \int_{0}^{N T}|s(t)|^{2} d t}
$$

Reducing the $\max |s(t)|$ is the principle goal of PARP reduction techniques. The PAPR reduction capability is measured by the empirical complementary cumulative distributive function (CCDF), which indicates the probability that the PAPR is above a certain threshold. Complementary Cumulative Distribution Function (CCDF) curves present vital information regarding the OFDM signal to be transmitted. The main use of power CCDF curves is to identify the power characteristic of the signals which are amplified, mixed and decoded [10] i.e. to find the probability of the PAPR. The ratio between power level and the average power is expressed in $\mathrm{db}$. For a transmitted $s(t)$ OFDM signal CCDF of $\{P A P R\{s(t)>\gamma\}\}$ is given as:

$$
\operatorname{Prob}(\operatorname{PAPR}\{s(t)\}>\gamma)=1-\left(1-e^{-\gamma}\right)^{N}
$$

where $N$ is the number of subcarriers.

\section{Clipping Technique}

The clipping [8] technique employs clipping around the peaks to reduce the PAPR. It is simple to implement. It reduces the PAPR by simply limiting the maximum amplitude of the OFDM signal, such that all signal values are limited to the threshold. Clipping the OFDM signal before amplification is a simple method to limit PAPR. The clipping operation is carried out at the transmitter. The clipping operation on the real band pass signal is given by: 
International Journal of Wireless \& Mobile Networks (IJWMN) Vol. 5, No. 1, February 2013

$$
k(t)=\left\{\begin{array}{c}
-A ; \quad \text { if } s(t) \leq-A \\
s(t) ; \quad \text { if }-A \leq s(t) \leq A \\
A ; \quad \text { if } s(t) \geq A
\end{array}\right\}
$$

where $k(t)$ is the clipped signal and $A$ is the clipping level. After which the exceeded signal was clipped.

Figure 2(a) shows the plot of an OFDM signal having maximum amplitude of 0.8. The peak of the OFDM signal exceeds the value from 0.6.This exceeded peak of the signal causes PAPR.

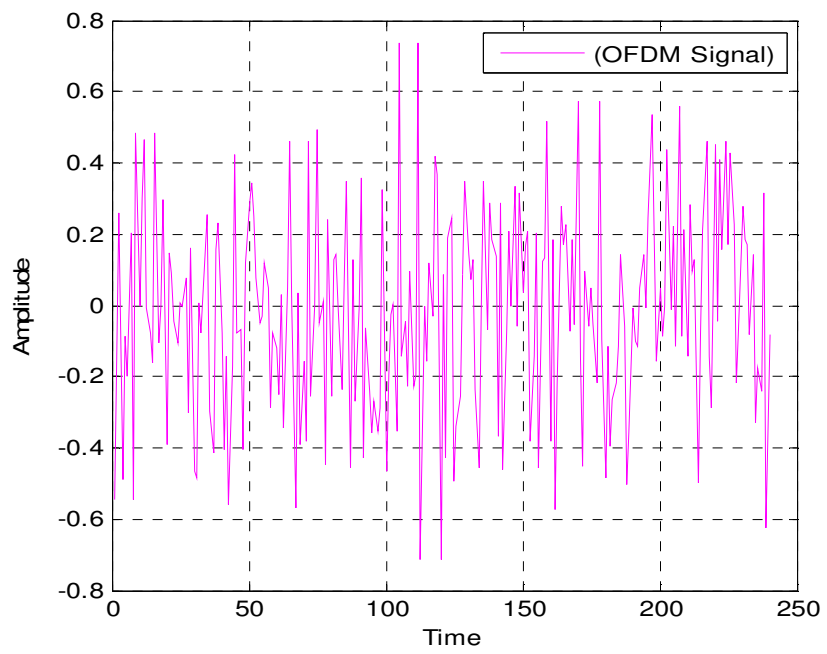

Figure 2(a). OFDM Signal with high peak value

To reduce the peak a threshold value was assumed in Figure 2(b) the value of the signal exceeding the value of 0.5 and -0.5 was clipped off. This reduction in the peak value limits the PAPR in the system.

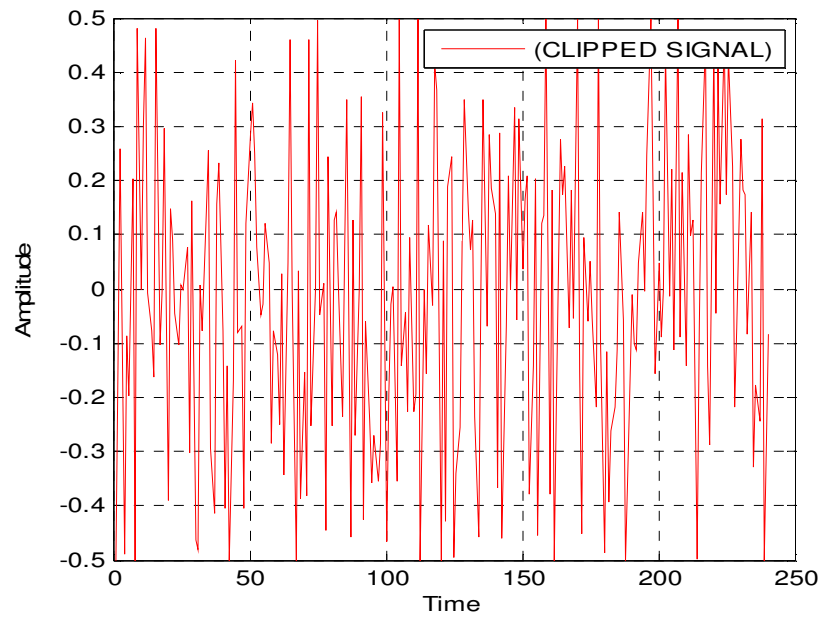

Figure 2(b).OFDM Signal with clipped peak value 
International Journal of Wireless \& Mobile Networks (IJWMN) Vol. 5, No. 1, February 2013

\section{WALSh HADAMARD TRANSFORM TEChNiQUE}

The goal of precoding techniques is to obtain a signal with lower PAPR than in the case of OFDM without precoding techniques and to reduce the interference produced by multiple users. The PAPR reduction must compensate the non linearities of the HPA having as effect the reduction of the bit error rate (BER). The main characteristics of precoding based techniques are: no bandwidth expansion, no power increase, and no data rate loss, no BER degradation and distortion less. WHT precoding technique is presented in the following.

The technique of Hadamard Transform [7] is based upon the relationship between correlation property of OFDM input sequence and PAPR probability. Theaverage power of the input sequence represents the peak value of the autocorrelation. Hence the peak value of autocorrelation depends on the input sequence provided thatnumber of sub carriers remains unchanged.

The Walsh Hadamard Transform (WHT) is a non-sinusoidal, orthogonal linear transform and can be implemented by a butterfly structure as in FFT. This means that applying WHT does not require the extensive increase of system complexity. WHT decomposes a signal into set of basic functions. These functions are Walsh functions, which are square waves with values of +1 or -1 [11]. The proposed hadamard transform scheme may reduce the occurrence of the high peaks comparing the original OFDM system. The idea to use the WHT is to reduce the autocorrelation of the input sequence to reduce the peak to average power problem and it requires no side information to be transmitted to the receiver. The kernel of WHT can be written as follows:

$$
\begin{aligned}
& \mathrm{H}_{1}=\text { [1] } \\
& \mathrm{H}_{2}=\frac{1}{\sqrt{2}}\left[\begin{array}{cc}
1 & 1 \\
1 & -1
\end{array}\right] \\
& \mathrm{H}_{2 \mathrm{~N}}=\frac{1}{\sqrt{2 \mathrm{~N}}}\left[\begin{array}{cc}
\mathrm{H}_{\mathrm{N}} & \mathrm{H}_{\mathrm{N}} \\
\mathrm{H}_{\mathrm{N}} & \mathrm{H}_{\mathrm{N}}^{-1}
\end{array}\right]
\end{aligned}
$$

where $H_{N}^{-1}$ denotes the binary complement of $H_{N}$.between each.

\section{Proposed SCHEME}

In the proposed scheme two appropriate methods are combined to reduce PAPR. One is the existing technique called clipping and the other is the precoding technique called walsh hadamard transform. The proposed combined peak clipping is different than normal peak clipping method because in normal peak clipping only one peak of OFDM symbol is clipped, whereas in proposed combined method PAPR reduces to much extent than by simple clipping The pre-coding has been considered as a best among all these techniques because it improves PAPR without increasing much complexity and without destroying the orthogonality between subcarriers. The combined techniques based OFDM system was shown in fig. 3. In proposed scheme conventional clipping was processed after WHT technique. At the transmitter end, the data stream is firstly transformed by WHT, and then the transformed data is processed by the clipping unit. If data block passed by WHT, before IFFT, the PAPR is reduced, then the PAPR of OFDM signal could be further reduced by clipping. 
International Journal of Wireless \& Mobile Networks (IJWMN) Vol. 5, No. 1, February 2013

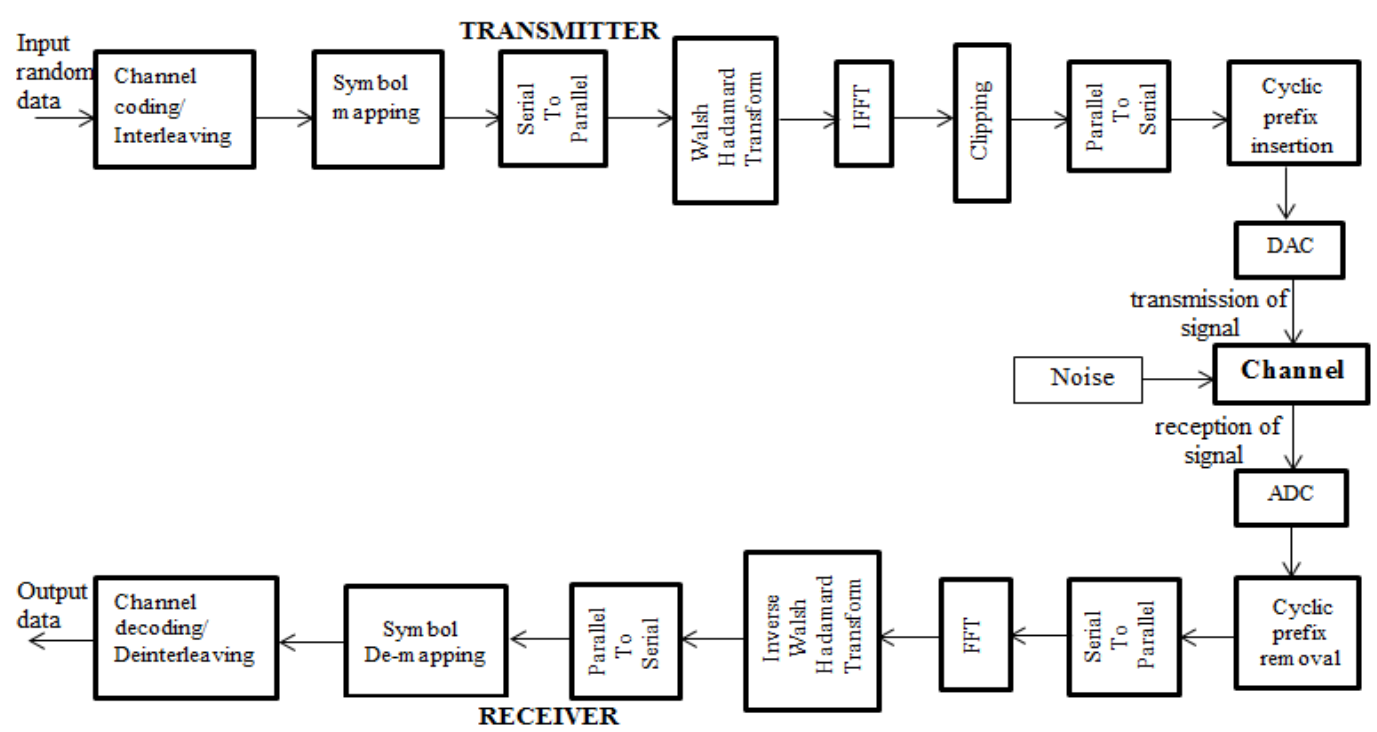

Figure 3. Block scheme of WHT Precoding technique with clipping in OFDM system

WHT precoding based OFDM system was shown in fig. 3. In these system, the kernel of the WHT acts as a precoding matrix Kof dimension $P=L \times L$ and it is applied to constellations symbols before the IFFT to reduce the correlation among the input sequence. In the precoding based systems baseband modulated data is passed through $\mathrm{S} / \mathrm{P}$ converter which generates a complex vector of size $L$ that can be written as $D=\left[D_{0}, D_{1}, \ldots, D_{L-1}\right]^{T}$.Then precoding is applied to this complex vector which transforms this complex vector into new vector of length $L$ that can be written as $b_{n}=K D=\left[b_{0}, b_{1}, \ldots, b_{L-1}\right]^{T}$ where $P$ is a precoder matrix of size $N=L \times L$ and $b_{n}$ can be written as follows:

$$
b_{n}=\sum_{l=0}^{L-1} K_{m, l} . D_{l} \quad ; m=0,1, \ldots, L-1
$$

$K_{m, l}$ means $m^{\text {th }}$ row and $l^{\text {th }}$ column of precoder matrix. The complex baseband OFDM signal with $N$ subcarriers can be written as:

$$
s(t)=\frac{1}{\sqrt{N}} \sum_{m=0}^{N-1} b_{n} \cdot e^{j 2 \pi m \frac{n}{N}} ; n=0,1,2, \ldots, N-1
$$

Clipping is performed on the I and Q outputs of the IFFT after WHT precoding was applied on OFDM signal. As the word length at the IFFT output is decreased, the power consumption and complexity of the DAC/ADC decreases. Also clipping at the IFFT output increases the resolution giving a better average signal.

\section{SimUlATION RESULTS}

In this section, the PAPR of OFDM with WHT precoding technique has been evaluated by simulation. To show PAPR analysis of the proposed system, the data is generated randomly then the signal is modulated by M-PSK and M-QAM respectively. The block implementation is shown in Fig. 3, where the precoding matrix transform represents proposed walsh hadamard transform precoding technique used in our simulations. We can evaluate the performance of the PAPR reduction scheme using the complementary cumulative distribution $(C C D F=$ $\operatorname{Prob}(\operatorname{PAPR}\{s(t)\}>\gamma)$ of the PAPR of the OFDM signal. The CCDF of the PAPR for WHT precoded OFDM signal is used to express the probability of exceeding a given threshold. We compared the simulation results of proposed system with WHT precoded OFDM systems and conventional OFDM systems. 


\subsection{M-PSK Modulation}

In this section WHT technique combined with clipping is applied over OFDM system with phase shift keying modulation (PSK).We assume CCDF clip rate of $10^{-0.7}$ using M-ary PSK technique for subcarriers $\mathrm{N}=2400$ and for different values of $\mathrm{M}$ results were evaluated where $\mathrm{M}=16,32,64,128,256,512$ and 1024.

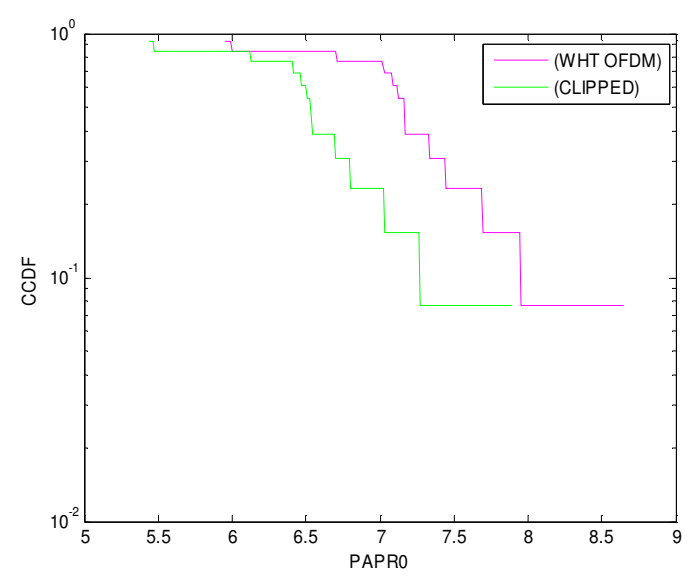

Figure 4. CCDF of clipping with proposed WHT technique for 16 PSK

For $\mathrm{M}=16$ for PSK modulation, the CCDF performance of the proposed clipped scheme compared with that of the WHT precoded technique for OFDM signal was shown in Figure 4.At CCDF clip rate of $10^{-0.7}$ the PAPR value of clipped precoded signal reduces by $0.75 \mathrm{~dB}$ over WHT precoded system.

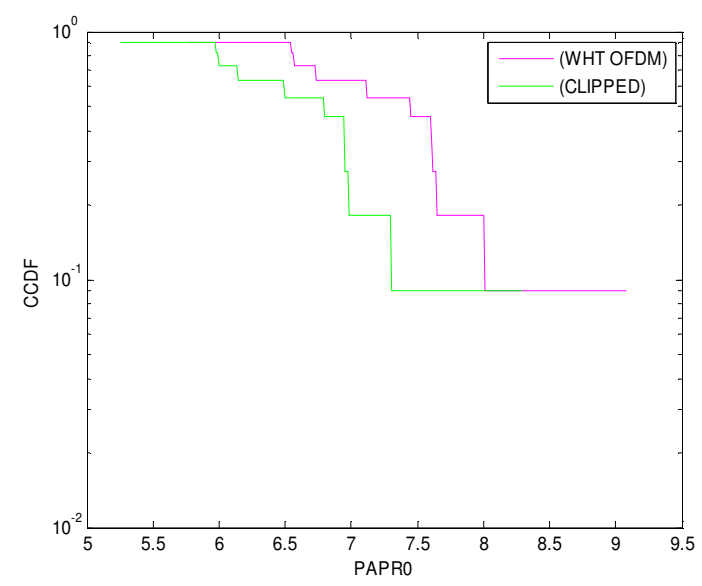

Figure 5. CCDF of clipping with proposed WHT technique for 32 PSK

For $\mathrm{M}=32$ for PSK modulation, the CCDF performance of the proposed clipped scheme compared with that of the WHT precoded technique for OFDM signal was shown in Figure 5. At CCDF clip rate of $10^{-0.7}$ the PAPR value of clipped precoded signal reduces by $0.72 \mathrm{~dB}$ over WHT precoded system. 


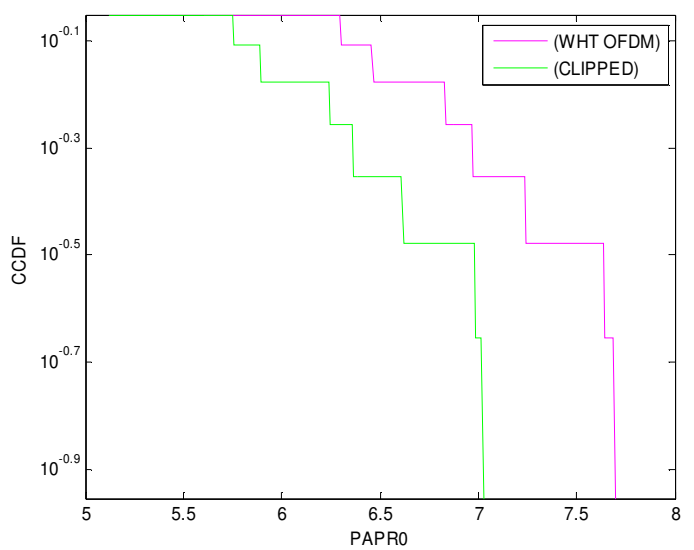

Figure 6. CCDF of clipping with proposed WHT technique for 64 PSK

For $\mathrm{M}=64$ for PSK modulation, the CCDF performance of the proposed clipped scheme compared with that of the WHT precoded technique for OFDM signal was shown in Figure 6. At CCDF clip rate of $10^{-0.7}$ the PAPR value of clipped precoded signal reduces by $0.69 \mathrm{~dB}$ over WHT precoded system.

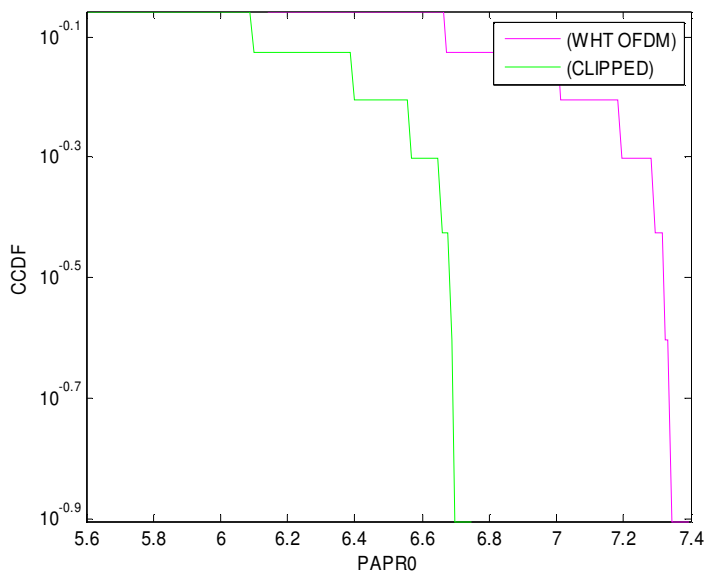

Figure 7. CCDF of clipping with proposed WHT technique for 128 PSK

For $\mathrm{M}=128$ for PSK modulation, the CCDF performance of the proposed clipped scheme compared with that of the WHT precoded technique for OFDM signal was shown in Figure 7. At CCDF clip rate of $10^{-0.7}$ the PAPR value of clipped precoded signal reduces by $0.67 \mathrm{~dB}$ over WHT precoded system. 
International Journal of Wireless \& Mobile Networks (IJWMN) Vol. 5, No. 1, February 2013

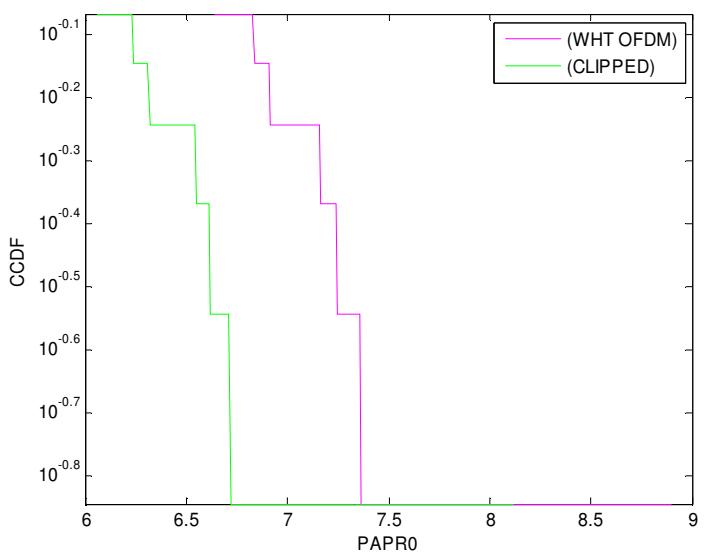

Figure 8. CCDF of clipping with proposed WHT technique for 256 PSK

For $\mathrm{M}=256$ for PSK modulation, the CCDF performance of the proposed clipped scheme compared with that of the WHT precoded technique for OFDM signal was shown in Figure 8. At CCDF clip rate of $10^{-0.7}$ the PAPR value of clipped precoded signal reduces by $0.64 \mathrm{~dB}$ over WHT precoded system.

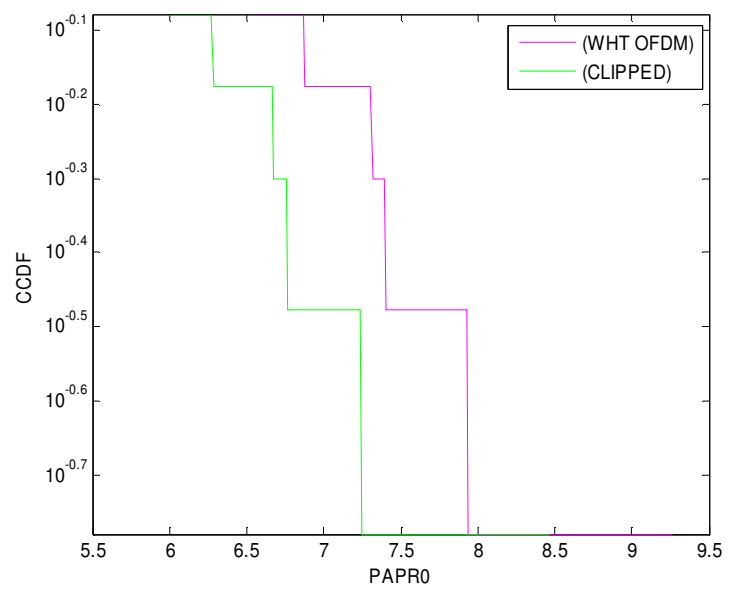

Figure 9. CCDF of clipping with proposed WHT technique for 512 PSK

For $\mathrm{M}=512$ for PSK modulation, the CCDF performance of the proposed clipped scheme compared with that of the WHT precoded technique for OFDM signal was shown in Figure 9. At CCDF clip rate of $10^{-0.7}$ the PAPR value of clipped precoded signal reduces by $0.62 \mathrm{~dB}$ over WHT precoded system. 
International Journal of Wireless \& Mobile Networks (IJWMN) Vol. 5, No. 1, February 2013

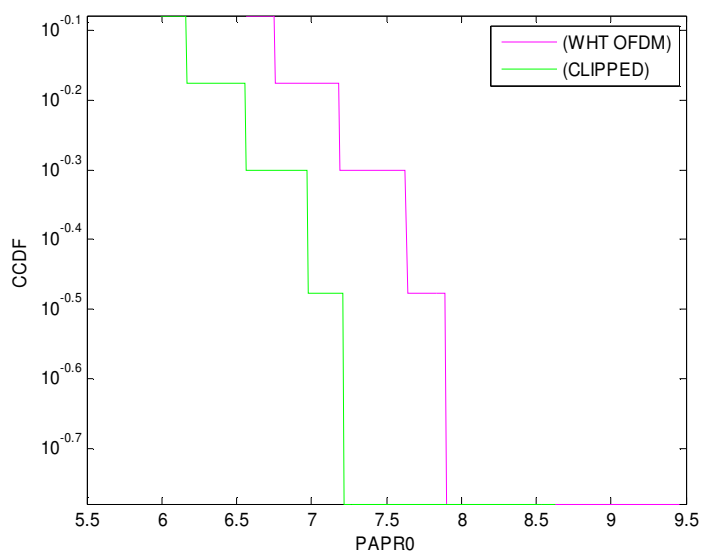

Figure 10. CCDF of clipping with proposed WHT technique for 1024 PSK

For $\mathrm{M}=1024$ for PSK modulation, the CCDF performance of the proposed clipped scheme compared with that of the WHT precoded technique for OFDM signal was shown in Figure 10. At CCDF clip rate of $10^{-0.7}$ the PAPR value of clipped precoded signal reduces by $0.61 \mathrm{~dB}$ over WHT precoded system.

\subsection{M-QAM Modulation}

In this section using M-ary quadrature amplitude modulation (QAM) technique is applied for $\mathrm{N}=2400$ and where $\mathrm{M}=16,64,256$ and 1024.

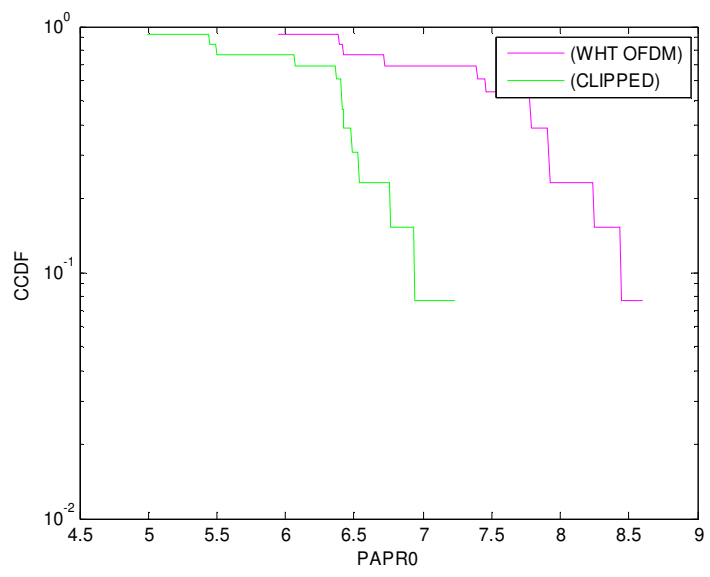

Figure 11. CCDF of clipping with proposed WHT technique for 16 QAM

For $\mathrm{M}=16$ for QAM modulation, the CCDF performance of the proposed clipped scheme compared with that of the WHT precoded technique for OFDM signal was shown in Figure 11. At CCDF clip rate of $10^{-0.7}$ the PAPR value of clipped precoded signal reduces by $1.6 \mathrm{~dB}$ over WHT precoded system. 
International Journal of Wireless \& Mobile Networks (IJWMN) Vol. 5, No. 1, February 2013

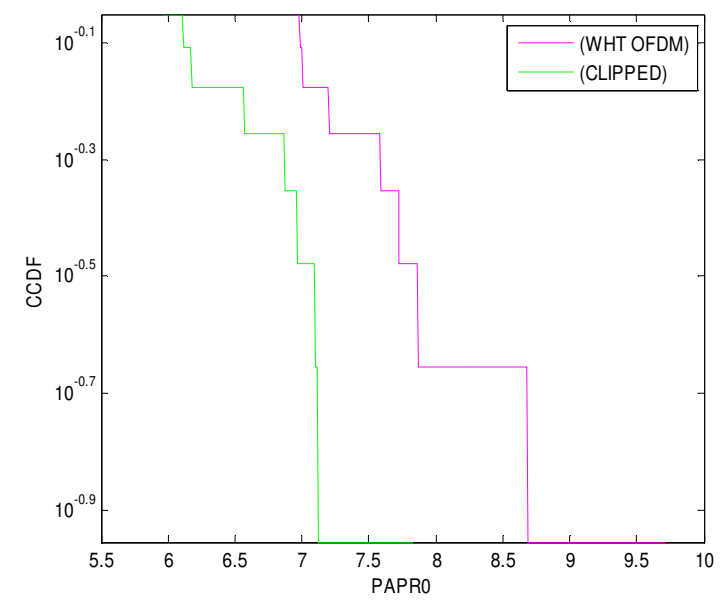

Fig. 12: CCDF of clipping with proposed WHT technique for 64 QAM

For $\mathrm{M}=64$ for $\mathrm{QAM}$ modulation, the CCDF performance of the proposed clipped scheme compared with that of the WHT precoded technique for OFDM signal was shown in Figure 12. At CCDF clip rate of $10^{-0.7}$ the PAPR value of clipped precoded signal reduces by $1.52 \mathrm{~dB}$ over WHT precoded system.

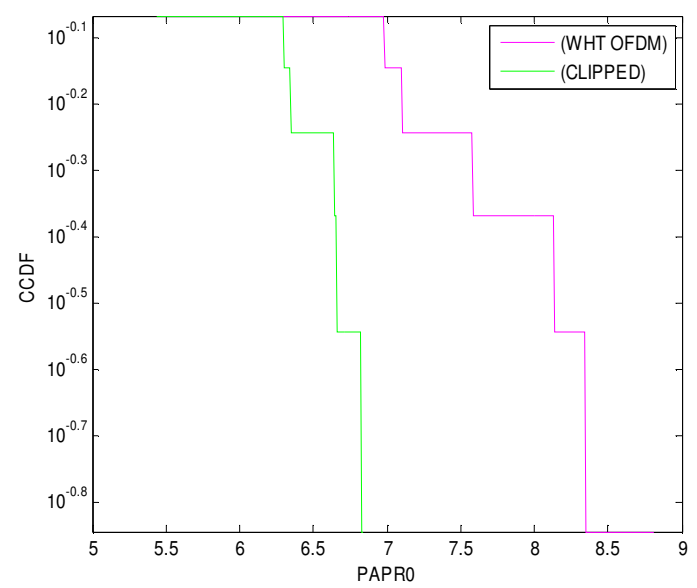

Fig. 13: CCDF of clipping with proposed WHT technique for 256 QAM

For $\mathrm{M}=256$ for $\mathrm{QAM}$ modulation, the CCDF performance of the proposed clipped scheme compared with that of the WHT precoded technique for OFDM signal was shown in Figure 13. At CCDF clip rate of $10^{-0.7}$ the PAPR value of clipped precoded signal reduces by $1.5 \mathrm{~dB}$ over WHT precoded system. 


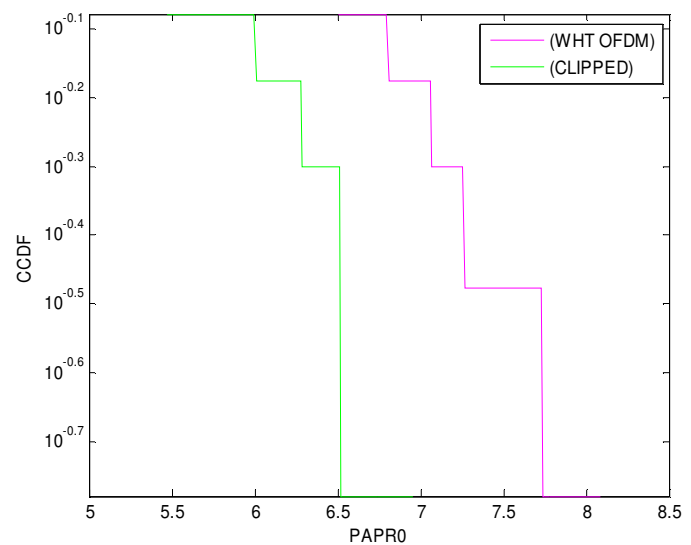

Fig. 14: CCDF of clipping with proposed WHT technique for 1024 QAM

For $\mathrm{M}=1024$ for QAM modulation, the CCDF performance of the proposed clipped scheme compared with that of the WHT precoded technique for OFDM signal was shown in Figure 14. At CCDF clip rate of $10^{-0.7}$ the PAPR value of clipped precoded signal reduces by $1.3 \mathrm{~dB}$ over WHT precoded system.

\section{CONCLUSIONS}

In this paper, to reduce PAPR in OFDM system, we evaluated performance of clipping with WHT precoding technique. The proposed combined technique is simple to implement and has no limitations on the system parameters such as number of subcarriers modulation order, and constellation type. This system produce the lowest PAPR and is efficient, signal independent, distortion less and do not require any complex optimizations representing better PAPR reduction methods than others existing techniques because it does not require any power increment, complex optimization and side information to be sent to the receiver.

From simulation results, it can be observed that the proposed combined clipped method displays a better PAPR reduction performance than the WHT precoded OFDM signal. Thus, it is concluded that the proposed combined clipped scheme is more favourable than the precoded WHT transform.

\section{ACKNOWLEDGEMENTS}

Foremost, I would like to express my sincere gratitude to Mr Lavish Kansal who gave his full support in the compilation of this report with his stimulating suggestions and encouragement to go ahead in all the time of the thesis. At last but not the least my gratitude towards my parents, I would also like to thank God for the strength that keep me standing and for the hope that keep me believing that this report would be possible.

\section{REFERENCES}

[1] R.W Chang, "Synthesis of Band-Limited Orthogonal Signals for Multi-channel Data Transmission," Bell System Technology, Vol.45, pp.1775-1797, 1966.

[2] R.W Chang, "Orthogonal Frequency Division Multiplexing," U.S Patent 3388455,1966.

[3] S .Weinstein \&P. Ebert, "Data Transmission by Frequency Division Multiplexing Using the Discrete Fourier Transform," IEEE Transaction on Communication, Vol.19, Issue: 5, pp. 628-634, 1971. 
International Journal of Wireless \& Mobile Networks (IJWMN) Vol. 5, No. 1, February 2013

[4] B.R. Satzberg, "Performance of an Efficient Parallel Data Transmission System,"IEEE Transaction on Communication, Vol. 15, Issue: 6, pp. 805-811, 1967.

[5] R. W. Bauml, R. F. Fischer \& J. B. Huber, "Reducing the Peak-to-Average Power Ration of Multicarrier Modulation by Selected Mapping," Electronics Letters, Vol. 32,Issue: 22, pp. 20502057, 1996.

[6] S. H. Muller \& J. B. Huber, "OFDM with Reduced Peak to Average Power Ratio by Optimum Combination of Partial Transmit Sequences," IEEE Electronics Letters, Vol. 33, Issue5, pp. 368369, 1997.

[7] M. Park, J. Heeyong, J. Cho, N. Cho, D. Hong \& C. Kang, "PAPR Reduction in OFDM Transmission Using Hadamard Transform," IEEE International Conference on Communications, Vol. 1, pp. 430-433, 2000.

[8] T. Jiang \& Y. Wu, “An Overview: Peak to Average Power Ratio Reduction Techniques for OFDM Signals,” IEEE Transactions on Broadcasting, Vol. 54, Issue: 2, pp. 257-268, 2008.

[9] S. H. Han \&J. H. Lee, "An Overview of Peak-to-Average Power Ratio Reduction Techniques for Multicarrier Transmission," IEEE Transactions on Wireless Communications, Vol. 12,Issue: 2, April 2005, pp. 56-65, 2005.

[10] Agilent Technologies Application Note, "Characterizing Digitally Modulated Signal with CCDF Curves", 2000.

[11] H. Rohling, Broadband OFDM Radio Transmission for Multimedia Applications, In IEEE Proceeding on Vehicular Conference, Vol. 87, Issue: 10, pp.1778-1788, 1999.

[12] L. Kansal, A. Kansal \& K. Singh, "Analysis of Different High Level Modulation Techniques for OFDM System,” International Journal of VLSI and Signal Processing Applications, Vol. 1, Issue: 2, 2011.

[13] L. Kansal, A. Kansal \& K. Singh, "Performance Analysis of MIMO-OFDM System Using QOSTBC Code Structure for M-PSK," Signal Processing: An International Journal, Vol. 5, Issue: 2, 2011.

[14] N. Kaur\& L. Kansal, "Reducing the Peak to Average Power Ratio of OFDM Signals through Walsh Hadamard Transform," Global Journal of Researches in Engineering, Vol. 13, Issue: 1, 2013.

\section{Authors}

Navneet Kaur was born in Kapurthala .She has done her B.Tech degree in Electronics and Communication Engineering from LovelyProfessional University, Phagwara, India. She is currently pursuing her M.Tech degree from Lovely Professional University, Phagwara, India. Her research area includes Digital Communication \& Wireless Communication.

Lavish Kansal was born in Bathinda.He received his B.Tech degree in Electronics and Communication Engineering from PTU, Jalandhar in 2009 and M.E. degree in Electronics and Communication Engineering from Thapar University, Patiala in 2011. He is working as Assistant Professor in the department of Electronics and communication Engineering, Lovely Professional University, Phagwara, India. He has published 15 papers in International journals. His research area includes Digital Signal Processing, Digital Communication \& Wireless Communication.
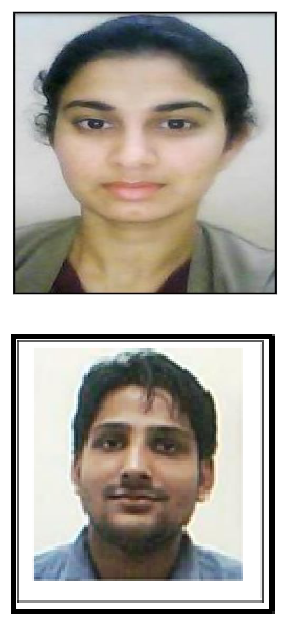